\title{
Ohlin-Type Theorem for Convex Set-Valued Maps
}

\author{
Kazimierz Nikodem(i) and Teresa Rajba
}

\begin{abstract}
A counterpart of the Ohlin theorem for convex set-valued maps is proved. An application of this result to obtain some inclusions related to convex set-valued maps in an alternative unified way is presented. In particular counterparts of the Jensen integral and discrete inequalities, the converse Jensen inequality and the Hermite-Hadamard inequalities are obtained.
\end{abstract}

Mathematics Subject Classification. Primary 26A51; Secondary 39B62, $26 \mathrm{D} 15$.

Keywords. Ohlin's lemma, convex set-valued maps, Jensen inequalities, Hermite-Hadamard inequalities.

\section{Introduction}

In Ohlin [12] proved the following interesting and very useful result on convex functions in a probabilistic context (as usual, $\mathbb{E}[X]$ denotes the expectation of the random variable $X)$ :

Lemma 1 [12]. Let $X_{1}, X_{2}$ be two real valued random variables such that $\mathbb{E}\left[X_{1}\right]=\mathbb{E}\left[X_{2}\right]$. If the distribution functions $F_{X_{1}}, F_{X_{2}}$ cross one time, i.e. there exists $t_{0} \in \mathbb{R}$ such that

$$
F_{X_{1}}(t) \leq F_{X_{2}}(t) \quad \text { if } t<t_{0} \text { and } F_{X_{1}}(t) \geq F_{X_{2}}(t) \quad \text { if } t>t_{0},
$$

then

$$
\mathbb{E}\left[f\left(X_{1}\right)\right] \leq \mathbb{E}\left[f\left(X_{2}\right)\right]
$$

for every convex function $f: \mathbb{R} \rightarrow \mathbb{R}$. 
For years the above Ohlin lemma was not well-known in the mathematical community. It has been rediscovered by Rajba [14], who found its various applications to the theory of functional inequalities. In $[13,15,18]$, the Ohlin lemma is used, among others, to get a simple proof of the known HermiteHadamard inequalities, as well as to obtaining new Hermite-Hadamard type inequalities.

In this note we prove counterparts of the Ohlin theorem for convex setvalued maps. We present also applications of these results to obtain some inclusions connected with convex set-valued maps.

\section{Preliminaries}

Let $(Y,\|\cdot\|)$ be a separable Banach space, $B$ be the closed unit ball in $Y$, $(\Omega, \mathcal{A}, P)$ be a probability space with a non-atomic measure $P$ and $I \subset \mathbb{R}$ be an open interval. Denote by $n(Y)$ the family of all nonempty subsets of $Y$ and by $c l(Y)$ the family of all closed nonempty subsets of $Y$. For a given set-valued map $G: \Omega \rightarrow n(Y)$ the integral $\int_{\Omega} G(\omega) d P$ is understood in the sense of Aumann, i.e. it is the set of integrals of all integrable (in the sense of Bochner) selections of the map $G$ (cf. $[1,2])$ :

$$
\int_{\Omega} G(\omega) d P=\left\{\int_{\Omega} g(\omega) d P: g: \Omega \rightarrow Y \quad \text { is integrable and } g(\omega) \in G(\omega), \omega \in \Omega\right\} .
$$

A set-valued map $G: \Omega \rightarrow n(Y)$ is called integrable bounded if there exists a non-negative integrable function $k: \Omega \rightarrow \mathbb{R}$ such that $G(\omega) \subset k(\omega) B$, for all $\omega \in \Omega$. In this case every measurable selection of $G$ is integrable and, consequently, the Aumann integral of $G$ is nonempty whenever $G$ is measurable.

The following properties of the Aumann integral will be needed in our investigations:

Lemma 2 [[1], Theorems 8.6.3, 8.6.4 ]. Let $G: \Omega \rightarrow c l(Y)$ be a measurable set-valued map. a) The closure of the integral of $G$ is convex and

$$
\overline{\int_{\Omega} G(\omega) d P}=\overline{\operatorname{conv}}\left(\int_{\Omega} G(\omega) d P\right) .
$$

b) If $Y$ is finite dimensional, then the integral of $G$ is convex. In particular, if $Y=\mathbb{R}$ and $G(\omega)=\left[g_{1}(\omega), g_{2}(\omega)\right], \omega \in \Omega$, then

$$
\int_{\Omega} G(\omega) d P=\left[\int_{\Omega} g_{1}(\omega) d P, \int_{\Omega} g_{2}(\omega) d P\right] .
$$

c) If $G$ is integrable bounded, then

$$
\overline{\int_{\Omega} G(\omega) d P}=\int_{\Omega} \overline{\operatorname{conv}} G(\omega) d P .
$$


Recall that a set-valued map $G: I \rightarrow n(Y)$ is called convex if

$$
t G\left(x_{1}\right)+(1-t) G\left(x_{2}\right) \subset G\left(t x_{1}+(1-t) x_{2}\right)
$$

for all $x_{1}, x_{2} \in I$ and $t \in[0,1]$ (see e.g. [1,3,4,8] and the references therein). Note that by (2), all values of $G$ are convex subsets of $Y$.

The following lemma characterizes convex set-valued maps with values in $\operatorname{cl}(\mathbb{R})$.

Lemma 3 [8] . A set-valued map $G: I \rightarrow c l(\mathbb{R})$ is convex if and only if it has one of the following forms:

a) $G(x)=\left[g_{1}(x), g_{2}(x)\right], \quad x \in I$,

b) $G(x)=\left[g_{1}(x),+\infty\right), \quad x \in I$

c) $G(x)=\left(-\infty, g_{2}(x)\right], \quad x \in I$,

d) $G(x)=(-\infty,+\infty), \quad x \in I$,

where $g_{1}: I \rightarrow \mathbb{R}$ is convex and $g_{2}: I \rightarrow \mathbb{R}$ is concave.

Clearly, if $G: I \rightarrow c l(\mathbb{R})$ is convex and integrable bounded, then it is of the form $a$ ).

\section{Ohlin-Type Result for Convex Set-Valued Maps}

The following result is a counterpart the Ohlin lemma for convex set-valued maps.

Theorem 4. Let $(Y,\|\cdot\|)$ be a separable Banach space, $(\Omega, \mathcal{A}, P)$ be a probability space with a non-atomic measure $P$ and $I \subset \mathbb{R}$ be an open interval. Assume that $X_{1}, X_{2}: \Omega \rightarrow I$ are integrable random variables such that $\mathbb{E}\left[X_{1}\right]=\mathbb{E}\left[X_{2}\right]$. If there exists $t_{0} \in \mathbb{R}$ such that

$$
F_{X_{1}}(t) \leq F_{X_{2}}(t) \quad \text { if } t<t_{0} \text { and } F_{X_{1}}(t) \geq F_{X_{2}}(t) \quad \text { if } t>t_{0},
$$

then

$$
\int_{\Omega} G\left(X_{2}(\omega)\right) d P \subset \int_{\Omega} G\left(X_{1}(\omega)\right) d P
$$

for every convex integrable bounded set-valued map $G: I \rightarrow \operatorname{cl}(Y)$.

Proof. The proof is divided into two steps. First, we assume that $Y=\mathbb{R}$. Then, by Lemma 3 and the assumption that $G$ is integrable bounded, we obtain that $G$ is of the form $G(x)=\left[g_{1}(x), g_{2}(x)\right], x \in I$, where $g_{1}: I \rightarrow \mathbb{R}$ is convex and $g_{2}: I \rightarrow \mathbb{R}$ is concave. By the Ohlin lemma (Lemma 1 ), we have

$$
\mathbb{E}\left[g_{1}\left(X_{1}\right)\right] \leq \mathbb{E}\left[g_{1}\left(X_{2}\right)\right] \text { and } \mathbb{E}\left[g_{2}\left(X_{1}\right)\right] \geq \mathbb{E}\left[g_{2}\left(X_{2}\right)\right]
$$

Hence, using Lemma 2(b), we get

$$
\begin{aligned}
\int_{\Omega} G\left(X_{2}(\omega)\right) d P & =\left[\mathbb{E}\left[g_{1}\left(X_{2}\right)\right], \mathbb{E}\left[g_{2}\left(X_{2}\right)\right]\right] \subset\left[\mathbb{E}\left[g_{1}\left(X_{1}\right)\right], \mathbb{E}\left[g_{2}\left(X_{1}\right)\right]\right] \\
& =\int_{\Omega} G\left(X_{1}(\omega)\right) d P .
\end{aligned}
$$


Now, assume that $Y$ is an arbitrary separable Banach space. Take a nonzero continuous linear functional $y^{*} \in Y^{*}$. Since the set-valued map $x \mapsto$ $\overline{y^{*}(G(x))}, x \in I$, is convex and has closed values in $\mathbb{R}$, by the previous step,

$$
\int_{\Omega} \overline{y^{*}\left(G\left(X_{2}(\omega)\right)\right)} d P \subset \int_{\Omega} \overline{y^{*}\left(G\left(X_{1}(\omega)\right)\right)} d P .
$$

Take arbitrary $z \in \int_{\Omega} G\left(X_{2}(\omega)\right) d P$. By the definition of the Aumann integral, there exists an integrable selection $g \circ X_{2}$ of the set-valued map $G \circ X_{2}$ such that $z=\int_{\Omega} g\left(X_{2}(\omega)\right) d P$. Using (4), we obtain

$$
y^{*}(z)=y^{*}\left(\int_{\Omega} g\left(X_{2}(\omega)\right) d P\right)=\int_{\Omega} y^{*}\left(g\left(X_{2}(\omega)\right)\right) d P \in \int_{\Omega} \overline{y^{*}\left(G\left(X_{1}(\omega)\right)\right)} d P .
$$

Since $G$ is integrable bounded and the values $y^{*}\left(G\left(X_{1}(\omega)\right)\right)$ are convex, by Lemma 2(c), we get

$$
\begin{aligned}
\int_{\Omega} \overline{y^{*}\left(G\left(X_{1}(\omega)\right)\right)} d P & =\int_{\Omega} \overline{\operatorname{conv}} y^{*}\left(G\left(X_{1}(\omega)\right)\right) d P=\overline{\int_{\Omega} y^{*}\left(G\left(X_{1}(\omega)\right) d P\right)} \\
& =\overline{y^{*}\left(\int_{\Omega} G\left(X_{1}(\omega)\right) d P\right)} \subset \overline{y^{*}\left(\overline{\left.\int_{\Omega} G\left(X_{1}(\omega)\right) d P\right)}\right.} .
\end{aligned}
$$

From (5) and (6),

$$
y^{*}(z) \in \overline{y^{*}\left(\overline{\int_{\Omega} G\left(X_{1}(\omega)\right) d P}\right)} .
$$

Since this condition holds for arbitrary $y^{*} \in Y^{*}$ and, by Lemma 2(a) the set $\overline{\int_{\Omega} G\left(X_{1}(\omega)\right) d P}$ is convex and closed, by the separation theorem (see [16], Corollary 2.5.11), we obtain

$$
z \in \overline{\int_{\Omega} G\left(X_{1}(\omega)\right) d P}
$$

and hence, using once more Lemma 2(c),

$$
z \in \int_{\Omega} G\left(X_{1}(\omega)\right) d P
$$

Consequently,

$$
\int_{\Omega} G\left(X_{2}(\omega)\right) d P \subset \int_{\Omega} G\left(X_{1}(\omega)\right) d P
$$

which finishes the proof.

Remark 5. In the above proof we use the Ohlin lemma (Lemma 1) to obtain the inequalities (3). Replacing in Theorem 4 the assumptions on $X_{1}$ and $X_{2}$ (the same as in Ohlin's lemma) by any weaker conditions sufficient for (3) (for instance necessary and sufficient conditions such as in the Levin-Stečkin theorem [7]; cf. also [11]), we can obtain more general result. However, it should 
be emphasized that the assumptions in the Ohlin lemma are very convenient because they are simple and can be easy verified.

\section{Applications}

In this section, we present an application of the Ohlin-type lemma to obtain various inclusions related to convex set-valued maps in a simple and unified way. Some of these results (Corollaries 6-10) are known, but we present alternative proofs of them.

The first result is a counterpart of the classical integral Jensen inequality.

Corollary 6 (cf. [8]). Let $G: I \rightarrow \operatorname{cl}(Y)$ be integrable bounded set-valued map and $(\Omega, \mathcal{A}, P)$ be a probability space with a non-atomic measure $P$. Then $G$ is convex if and only if

$$
\int_{\Omega} G(X(\omega)) d P \subset G\left(\int_{\Omega} X(\omega) d P\right),
$$

for every integrable random variable $X: \Omega \rightarrow I$.

Proof. Assume first that $G: I \rightarrow \operatorname{cl}(Y)$ is a convex integrable bounded setvalued map and $X: \Omega \rightarrow I$ is an integrable random variable. Take a random variable $X_{1}: \Omega \rightarrow I$ with the distribution $\mu_{X_{1}}=\delta_{\mathbb{E}[X]}$. Then the distribution functions $F_{X}, F_{X_{1}}$ satisfy condition (1) and $\mathbb{E}[X]=\mathbb{E}\left[X_{1}\right]$. Therefore, by Theorem 4,

$$
\int_{\Omega} G(X(\omega)) d P \subset \int_{\Omega} G\left(X_{1}(\omega)\right) d P=G(\mathbb{E}[X])=G\left(\int_{\Omega} X(\omega) d P\right) .
$$

Now, assume that $G$ satisfies condition (7) with every integrable random variable $X: \Omega \rightarrow I$. Fix $x_{1}, x_{2} \in I$ and $t \in(0,1)$, and take a random variable $X: \Omega \rightarrow I$ with the distribution $\mu_{X}=t \delta_{x_{1}}+(1-t) \delta x_{2}$. Then $\int_{\Omega} X(\omega) d P=$ $t x_{1}+(1-t) x_{2}$ and $\int_{\Omega} G(X(\omega)) d P=t G\left(x_{1}\right)+(1-t) G\left(x_{2}\right)$. Therefore by $(7)$

$$
t G\left(x_{1}\right)+(1-t) G\left(x_{2}\right) \subset G\left(t x_{1}+(1-t) x_{2}\right),
$$

which proves that $G$ is convex.

If in the above corollary we take a random variable $X$ with the distribution $\mu_{X}=t_{1} \delta_{x_{1}}+\cdots+t_{n} \delta_{x_{n}}$, where $x_{1}, \ldots, x_{n} \in I$ and $t_{1}, \ldots, t_{n}>0$ are such that $t_{1}+\cdots+t_{n}=1$, then we obtain a counterpart of the discrete Jensen inequality.

Corollary 7 (cf. [10]). If a set-valued map $G: I \rightarrow \operatorname{cl}(Y)$ is convex and integrable bounded, then

$$
\sum_{i=1}^{n} t_{i} G\left(x_{i}\right) \subset G\left(\sum_{i=1}^{n} t_{i} x_{i}\right)
$$

for all $n \in \mathbb{N}, x_{1}, \ldots, x_{n} \in I$ and $t_{1}, \ldots, t_{n}>0$ with $t_{1}+\cdots+t_{n}=1$. 
We have also the following converse Jensen inclusion for convex set-valued maps.

Corollary 8 (cf. [6]). Let $m, M \in I, m<M$. If $G: I \rightarrow \operatorname{cl}(Y)$ is convex and integrable bounded, then

$$
\frac{M-\bar{x}}{M-m} G(m)+\frac{\bar{x}-m}{M-m} G(M) \subset \sum_{i=1}^{n} t_{i} G\left(x_{i}\right),
$$

for all $x_{1}, \ldots, x_{n} \in[m, M], t_{1}, \ldots, t_{n}>0$ with $t_{1}+\cdots+t_{n}=1$ and $\bar{x}=$ $t_{1} x_{1}+\cdots+t_{n} x_{n}$.

Proof. Take random variables $X_{1}, X_{2}: \Omega \rightarrow I$ with the distributions

$$
\mu_{X_{1}}=\sum_{i=1}^{n} t_{i} \delta_{x_{i}} \text { and } \mu_{X_{2}}=\frac{M-\bar{x}}{M-m} \delta_{m}+\frac{\bar{x}-m}{M-m} \delta_{M} .
$$

Then the distribution functions $F_{X}, F_{Y}$ satisfy condition (1) and

$$
\mathbb{E}\left[X_{1}\right]=\sum_{i=1}^{n} t_{i} x_{i}=\bar{x}=\frac{M-\bar{x}}{M-m} m+\frac{\bar{x}-m}{M-m} M=\mathbb{E}\left[X_{2}\right] .
$$

Moreover

$$
\int_{\Omega} G\left(X_{1}(\omega)\right) d P=\sum_{i=1}^{n} t_{i} G\left(x_{i}\right)
$$

and

$$
\int_{\Omega} G\left(X_{2}(\omega)\right) d P=\frac{M-\bar{x}}{M-m} G(m)+\frac{\bar{x}-m}{M-m} G(M) .
$$

Therefore, by Theorem 4, we obtain (8).

The next two corollaries are versions of the Hermite-Hadamard inequalities for convex set-valued maps.

Corollary 9 (cf. $[9,17]$ ). If $G: I \rightarrow c l(Y)$ is convex and integrable bounded, then

$$
\frac{G(a)+G(b)}{2} \subset \frac{1}{b-a} \int_{a}^{b} G(x) d x \subset G\left(\frac{a+b}{2}\right),
$$

for all $a, b \in I, a<b$.

Proof. Let $X_{1}, X_{2}: \Omega \rightarrow I$ be random variables with the distributions $\mu_{X_{1}}=$ $\delta_{(a+b) / 2}, \mu_{X_{2}}=\frac{1}{2}\left(\delta_{a}+\delta_{b}\right)$ and let $X_{3}: \Omega \rightarrow I$ has the uniform distribution on $[a, b]$. Then the pairs $X_{1}, X_{3}$ and $X_{3}, X_{2}$ satisfy the assumptions of Theorem 4. Moreover,

$$
\int_{\Omega} G\left(X_{1}(\omega)\right) d P=G\left(\frac{a+b}{2}\right), \int_{\Omega} G\left(X_{2}(\omega)\right) d P=\frac{G(a)+G(b)}{2}
$$

and

$$
\int_{\Omega} G\left(X_{3}(\omega)\right) d P=\frac{1}{b-a} \int_{a}^{b} G(x) d x .
$$

Therefore, by Theorem 4, we obtain (9). 
Corollary 10. (cf. [9]) If $G: I \rightarrow c l(Y)$ is convex and integrable bounded, $[a, b] \subset I$ and $\mu$ is a Borel measure on $[a, b]$ with $\mu([a, b])>0$, then

$$
\frac{b-x_{\mu}}{b-a} G(a)+\frac{x_{\mu}-a}{b-a} G(b) \subset \frac{1}{\mu([a, b])} \int_{a}^{b} G(x) d \mu(x) \subset G\left(x_{\mu}\right),
$$

where $x_{\mu}=\frac{1}{\mu([a, b])} \int_{a}^{b} x d \mu(x)$ is the barycenter of $\mu$ on $[a, b]$.

Proof. By the mean value theorem $x_{\mu} \in[a, b]$. Let $X_{1}, X_{2}, X_{3}: \Omega \rightarrow[a, b]$ be random variables with the distributions

$$
\mu_{X_{1}}=\delta_{x_{\mu}}, \quad \mu_{X_{2}}=\frac{b-x_{\mu}}{b-a} \delta_{a}+\frac{x_{\mu}-a}{b-a} \delta_{b}, \quad \mu_{X_{3}}=\frac{1}{\mu([a, b])} \mu .
$$

Then the pairs $X_{1}, X_{3}$ and $X_{3}, X_{2}$ satisfy the assumptions of Theorem 4 . Moreover,

$$
\int_{\Omega} G\left(X_{1}(\omega)\right) d P=G\left(x_{\mu}\right), \int_{\Omega} G\left(X_{2}(\omega)\right) d P=\frac{b-x_{\mu}}{b-a} G(a)+\frac{x_{\mu}-a}{b-a} G(b)
$$

and

$$
\int_{\Omega} G\left(X_{3}(\omega)\right) d P=\frac{1}{\mu([a, b])} \int_{a}^{b} G(x) d \mu(x) .
$$

Therefore, by Theorem 4, we obtain (10).

The next two corollaries are counterparts for convex set-valued maps of the following inequalities concerning convex functions $f: I \rightarrow \mathbb{R}(\mathrm{cf}$. $[5,15])$ :

$$
\frac{f(c)+f(d)}{2}-f\left(\frac{c+d}{2}\right) \leq \frac{f(a)+f(b)}{2}-f\left(\frac{a+b}{2}\right)
$$

for all $a, b, c, d \in I$ such that $a<c<d<b$, and the Popoviciu inequality

$$
\begin{aligned}
\frac{2}{3}\left[f\left(\frac{x+y}{2}\right)+f\left(\frac{y+z}{2}\right)+f\left(\frac{z+x}{2}\right)\right] \leq & \frac{f(x)+f(y)+f(z)}{3} \\
& +f\left(\frac{x+y+z}{3}\right),
\end{aligned}
$$

for all $x, y, z \in I$.

Corollary 11. If $G: I \rightarrow \operatorname{cl}(Y)$ is convex and integrable bounded, then

$$
\frac{G(a)+G(b)}{2}+G\left(\frac{c+d}{2}\right) \subset \frac{G(c)+G(d)}{2}+G\left(\frac{a+b}{2}\right),
$$

for all $a, b, c, d \in I$ such that $a<c<d<b$.

Proof. Let $X_{1}, X_{2}: \Omega \rightarrow I$ be random variables with the distributions

$$
\mu_{X_{1}}=\frac{1}{4}\left(\delta_{c}+\delta_{d}\right)+\frac{1}{2} \delta_{(a+b) / 2}, \quad \mu_{X_{2}}=\frac{1}{4}\left(\delta_{a}+\delta_{b}\right)+\frac{1}{2} \delta_{\delta_{(c+d) / 2}} .
$$


Then the pair $X_{1}, X_{2}$ satisfies the assumptions of Theorem 4. Moreover,

$$
\begin{aligned}
& \int_{\Omega} G\left(X_{1}(\omega)\right) d P=\frac{G(c)+G(d)}{4}+\frac{1}{2} G\left(\frac{a+b}{2}\right), \\
& \int_{\Omega} G\left(X_{2}(\omega)\right) d P=\frac{G(a)+G(b)}{4}+\frac{1}{2} G\left(\frac{c+d}{2}\right) .
\end{aligned}
$$

Therefore, by Theorem 4, we obtain (11).

Corollary 12. If $G: I \rightarrow c l(Y)$ is convex and integrable bounded, then

$$
\begin{aligned}
\frac{G(x)+G(y)+G(z)}{3}+G\left(\frac{x+y+z}{3}\right) \subset & \frac{2}{3}\left[G\left(\frac{x+y}{2}\right)+G\left(\frac{y+z}{2}\right)\right. \\
& \left.+G\left(\frac{z+x}{2}\right)\right]
\end{aligned}
$$

for all $x, y, z \in I$.

Proof. Let $X_{1}, X_{2}: \Omega \rightarrow I$ be random variables with the distributions

$$
\begin{array}{r}
\mu_{X_{1}}=\frac{1}{3}\left(\delta_{(x+y) / 2}+\delta_{(y+z) / 2}+\delta_{(z+x) / 2}\right), \\
\mu_{X_{2}}=\frac{1}{6}\left(\delta_{x}+\delta_{y}+\delta_{z}\right)+\frac{1}{2} \delta_{(x+y+z) / 3} .
\end{array}
$$

Then the pair $X_{1}, X_{2}$ satisfies the assumptions of Theorem 4. Moreover,

$$
\begin{array}{r}
\int_{\Omega} G\left(X_{1}(\omega)\right) d P=\frac{1}{3}\left[G\left(\frac{x+y}{2}\right)+G\left(\frac{y+z}{2}\right)+G\left(\frac{z+x}{2}\right)\right] \\
\int_{\Omega} G\left(X_{2}(\omega)\right) d P=\frac{G(x)+G(y)+G(z)}{6}+\frac{1}{2} G\left(\frac{x+y+z}{3}\right) .
\end{array}
$$

Therefore, by Theorem 4, the corollary is proved.

Open Access. This article is licensed under a Creative Commons Attribution 4.0 International License, which permits use, sharing, adaptation, distribution and reproduction in any medium or format, as long as you give appropriate credit to the original author(s) and the source, provide a link to the Creative Commons licence, and indicate if changes were made. The images or other third party material in this article are included in the article's Creative Commons licence, unless indicated otherwise in a credit line to the material. If material is not included in the article's Creative Commons licence and your intended use is not permitted by statutory regulation or exceeds the permitted use, you will need to obtain permission directly from the copyright holder. To view a copy of this licence, visit http://creativecommons.org/ licenses/by $/ 4.0 /$. 


\section{References}

[1] Aubin, J.-P., Frankowska, H.: Set-Valued Analysis. Birkhäuser, Boston (1990)

[2] Aumann, R.J.: Integrals of set-valued functions. J. Math. Anal. Appl. 12, 1-12 (1965)

[3] Benoist, J., Popovici, N.: Generalized convex set-valued maps. J. Math. Anal. Appl. 288, 161-166 (2003)

[4] Borwein, J.M.: Multivalued convexity and optimization: a unified approach to inequality and equality constrains. Math. Program. 13, 183-199 (1977)

[5] Hardy, G.H., Littlewood, J.E., Pólya, G.: Inequalities. Cambridge University Press, Cambridge (1952)

[6] Klaričić Bakula, M., Nikodem, K.: Converse Jensen inequality for strongly convex set-valued maps. J. Math. Inequal. 12(2), 545-550 (2018)

[7] Levin, V.I., Stečkin, S.B.: Inequalities. Am. Math. Soc. Transl. 14, 1-22 (1960)

[8] Matkowski, J., Nikodem, K.: An integral Jensen inequality for convex multifunctions. Res. Math. 26, 348-353 (1994)

[9] Mitroi, F.-C., Nikodem, K., Wąsowicz, S.: Hermite-Hadamard inequalities for convex set-valued functions. Demonstr. Math. 46, 655-662 (2013)

[10] Nikodem, K.: $K$-convex and $K$-concave set-valued functions, Zeszyty Nauk. Politech. Łódz. Mat. 559 (Rozprawy Nauk 114), Łódź, pp. 1-75 (1989)

[11] Nikodem, K., Rajba, T.: Ohlin and Levin-Stečkin-type results for strongly convex functions. Ann. Math. Silesianae (2020). https://doi.org/10.2478/ amsil-2020-0017

[12] Ohlin, J.: On a class of measures of dispersion with application to optimal reinsurance. ASTIN Bull. 5, 249-266 (1969)

[13] Olbryś, A., Szostok, T.: Inequalities of the Hermite-Hadamard type involving numerical differentiation formulas. Res. Math. 67, 403-416 (2015)

[14] Rajba, T.: On the Ohlin lemma for Hermite-Hadamard-Fejér type inequalities. Math. Inequal. Appl. 17, 557-571 (2014)

[15] Rajba, T.: On some recent applications of stochastic convex ordering theorems to some functional inequalities for convex functions : a survey. In: Brzdȩk, J., Ciepliński, K., Rassias, T.M. (eds.) Developments in Functional Equations and Related Topics, vol. 124, 11th edn, pp. 231-274. Springer Optimizations and Its Applications, Berlin (2017)

[16] Rolewicz, S.: Functional Analysis and Control Theory. Linear Systems. PWN Polish Scientific Publishers, Dordrecht (1987)

[17] Sadowska, E.: Hadamard inequality and a refinement of Jensen inequality for set-valued functions. Res. Math. 32, 332-337 (1997)

[18] Szostok, T.: Ohlin's lemma and some inequalities of the Hermite-Hadamard type. Aequat. Math. 89, 915-926 (2015) 
Kazimierz Nikodem and Teresa Rajba

Department of Mathematics

University of Bielsko-Biala

ul. Willowa 2

43-309 Bielsko-Biała

Poland

e-mail: knikodem@ath.bielsko.pl;

trajba@ath.bielsko.pl

Received: April 17, 2020.

Accepted: October 1, 2020.

Publisher's Note Springer Nature remains neutral with regard to jurisdictional claims in published maps and institutional affiliations. 